\title{
Paramètres de découpe et de mensurations des carcasses d'agneaux Martinik élevés en conditions intensives
}

\author{
G. Alexandre ${ }^{1 *}$ B. Bocage ${ }^{2}$ O. Coppry ${ }^{3}$ \\ J.L. Weisbecker ${ }^{4}$ M. Mahieu ${ }^{1}$ H. Archimède ${ }^{1}$
}

Mots-clés

Ovin Martinik - Carcasse Mensuration corporelle Conformation animale Croissance - Antilles françaises.

\begin{abstract}
Résumé
L'ovin Martinik est sélectionné pour ses capacités d'adaptation et sa productivité. Les filières lui reprochent une faible conformation de carcasse, alors même que ces carcasses ont été peu décrites. Au cours de deux essais d'engraissement intensif, des abattages ont été réalisés et des découpes et des mensurations linéaires ont été relevées. Ces variables ont été analysées et la détermination des équations d'allométrie a été réalisée. La base de données ( $n=43$ agneaux) a été codifiée pour les niveaux d'alimentation et la classe de poids d'abattage (PA : 28, 32 et $36 \mathrm{~kg}$ ). Les poids moyens de carcasse froide (CF) ont varié $(\mathrm{P}<0,05)$ de 11,7 à $15,6 \mathrm{~kg}$. Les rendements de carcasse ont été en moyenne de 59 p. $100(P>0,05)$. Les poids des différents morceaux ont augmenté significativement $(\mathrm{P}<0,01)$ avec la gamme de CF. Les proportions d'épaule et de gigot ont représenté respectivement 18 et 34 p. 100 de la CF. Les différentes longueurs mesurées sur la carcasse et la largeur de bassin ont augmenté $(P<0,05)$ avec la CF (près de 9 p. 100 de différence entre les poids extrêmes). Les index de compacité de carcasse $(0,32)$ et de gigot $(0,45)$ n'ont pas varié selon le PA, alors que les index pondéraux ont été différents. Les coefficients d'allométrie de l'épaule $(0,915)$ et du gigot $(0,891)$ ont été du même ordre de grandeur que ceux de la littérature. Ces premières descriptions des carcasses de l'ovin Martinik pourraient être proposées à la filière afin de poursuivre le travail et mieux baser les choix de race et de systèmes.
\end{abstract}

\section{INTRODUCTION}

Aux Antilles françaises, l'élevage de moutons est basé sur l'exploitation de la race Ovin Martinik (OMK) qui est un génotype à poils (hair sheep, ovino de pelo) très répandu dans la Caraïbe (24). La sélection de l'OMK prend en compte ses capacités d'adaptation (résistance au climat et aux parasites), de reproduction (désaisonnement et prolificité) et ses qualités maternelles (31). Les performances d'engraissement et la conformation de la carcasse sont encore peu étudiées. Les éleveurs souhaitent alourdir les carcasses et les bouchers lui reprochent une faible conformation

1. Inra, UR 143 Recherches zootechniques, Petit Bourg, F-97170 Guadeloupe.

2. Inra, UE 503 Production et santé animale, Petit Bourg, F-97170 Guadeloupe.

3. Inra, UE 467, Domaine Gardel, Le Moule, F-97160 Guadeloupe.

4. Inra, UE0065 Génétique animale, Montgiscard, F-31450 France.

* Auteur pour la correspondance

Tél. : +590 (0)5 90255933 ; fax: +590 (0)5 90255936

E-mail : gisele.alexandre@antilles.inra.fr
[Association martiniquaise de l'interprofession viande (Amiv), et Interprofession guadeloupéenne de la viande et de l'élevage (Iguavie) ; commun. pers.]. Or, il est difficile de faire la part entre les facteurs génétiques et environnementaux $(12,29,32)$ dans l'obtention des performances.

$\mathrm{Au}$ vu des formats des races exploitées en Europe, l'agneau OMK est de la catégorie des agneaux légers (9-12 kg carcasse). Cependant, le mode d'élevage le plus répandu se déroule au pâturage (20) qui ne permet pas l'alourdissement des carcasses et l'amélioration de la conformation. Ceux-ci dépendent de l'ingestion d'aliments riches en énergie $(3,8,19)$. Quelques expérimentations ont été conduites en systèmes intensifs avec les moutons OMK $(1,5)$ afin de déterminer l'efficience alimentaire des rations et les résultats technico-économiques. Cependant, la description de la conformation des carcasses ainsi que la proportion des morceaux découpés, connues et utilisées depuis longtemps dans la filière viande française (7), ont été peu étudiées sous nos latitudes. Il est apparu nécessaire de fournir des données factuelles et de les partager avec les acteurs des filières Amiv et Iguavie. 
Au cours des deux essais d'engraissement intensif cités, l'un à base de canne à sucre et l'autre avec un gradient d'apports de concentrés, lors des abattages, des mensurations linéaires et pondérales ont été relevées en même temps que des notations subjectives de la conformation de la carcasse. L'étude a été complétée par la détermination des équations d'allométrie reliant les poids des morceaux à ceux du poids vif vide ou de la carcasse.

\section{MATERIEL ET METHODES}

\section{Conduite des animaux}

Les agneaux de race OMK ont été élevés en stabulation après sevrage durant près de quatre mois d'engraissement. Les conditions expérimentales détaillées sont décrites par Alexandre et coll. (1) pour l'essai 1, et Archimède et coll. (5) pour l'essai 2.

\section{Essai 1}

Quarante agneaux Martinik élevés en stabulation libre ont reçu une ration de canne à sucre entière broyée $[0,69$ unité fourragère lait (UFL) et $15 \mathrm{~g}$ de teneur en protéines digestibles dans l'intestin permises par l'azote de l'aliment (PDIN)/kg de matière sèche (MS)]. Le complément azoté a été apporté par des pois (Pisum sativum ; 1,16 UFL et $155 \mathrm{~g}$ PDIN/kg MS) à raison de 12-14 p. 100 de la ration. Les animaux ont pesé $20,2 \pm 3,0 \mathrm{~kg}$ à quatre mois en début de période d'adaptation.

\section{Essai 2}

Quatre lots d'agneaux (10 par lot) recevant du fourrage vert $(0,72$ UFL et $90 \mathrm{~g}$ PDIN/kg MS) ont été comparés sur la base de l'apport de concentrés $(1,15$ UFL et $112 \mathrm{~g}$ PDIN/kg MS) dans la ration : le lot L0 a reçu la ration de base sans concentrés, les lots L150, L300 et L600 ont reçu, en plus, respectivement 150, 300 et $600 \mathrm{~g}$ de concentrés par agneau et par jour. Les animaux ont pesé $20 \pm$ $3,7 \mathrm{~kg}$ à quatre mois en début d'essai.

\section{Mesures à l'abattage et sur carcasse}

Les animaux ont été abattus entre 24 et $38 \mathrm{~kg}$, et entre 26 et $38 \mathrm{~kg}$ respectivement dans l'expérimentation 1 et 2 (tableau I). La procédure classique a été mise en œuvre (abattage, saignée, éviscération, dépeçage). Les carcasses ont été placées durant $24 \mathrm{~h}$ de ressuyage en chambre froide à $4{ }^{\circ} \mathrm{C}$. Elles ont été pesées chaudes (CC) et froides $(\mathrm{CF})$. Sur la carcasse froide, une note de conformation (de 1 à 5) et des notes de gras interne et externe (de 1 à 5) ont été attribuées selon la méthode de routine adaptée aux agneaux légers (26).

Plusieurs mensurations linéaires ont été réalisées selon une méthode de routine (11) avec un pied à coulisse ou un mètre ruban : sur la carcasse entière pendue, plus grande largeur au niveau des trochanters et longueur de carcasse queue - cou ; après avoir scié la carcasse en deux parties égales le long de la colonne vertébrale, sur la demi-carcasse gauche pendue, longueur jarret -

\section{Tableau I}

Statistiques descriptives pour les variables de carcasse des agneaux Martinik conduits dans des expérimentations différentes

\begin{tabular}{|c|c|c|c|c|c|c|c|}
\hline Variable & Exp. ${ }^{1}$ & $\mathbf{n}$ & Moyenne & $\begin{array}{l}\text { Déviation } \\
\text { standard }\end{array}$ & $\begin{array}{l}\mathrm{CV}^{2} \\
(\%)\end{array}$ & Minimum & Maximum \\
\hline \multirow{3}{*}{$\begin{array}{l}\text { Gain de poids } \\
\text { moyen quotidien (g/j) }\end{array}$} & 1 & 20 & 116,7 & 26,6 & 23 & 57,1 & 168,6 \\
\hline & 2 & 23 & 172,4 & 30,8 & 18 & 124,4 & 224,8 \\
\hline & 3 & 44 & 78,4 & 16,5 & 21 & 42,0 & 115,0 \\
\hline \multirow[t]{3}{*}{ Poids à jeun (kg) } & 1 & 20 & 30,5 & 3,6 & 12 & 23,9 & 37,9 \\
\hline & 2 & 23 & 33,0 & 3,4 & 10 & 26,4 & 38,1 \\
\hline & 3 & 44 & 22,4 & 4,7 & 21 & 13,9 & 32,5 \\
\hline \multirow[t]{3}{*}{ Carcasse froide (kg) } & 1 & 20 & 13,8 & 1,9 & 14 & 10,2 & 17,8 \\
\hline & 2 & 23 & 13,5 & 1,7 & 13 & 9,2 & 17,0 \\
\hline & 3 & 44 & 9,3 & 2,1 & 22 & 5,6 & 14,4 \\
\hline \multirow[t]{3}{*}{ Epaule (g) } & 1 & 20 & 1228 & 169 & 14 & 890 & 1600 \\
\hline & 2 & 23 & 1211 & 121 & 10 & 1008 & 1422 \\
\hline & 3 & 44 & 960 & 114 & 12 & 770 & 1170 \\
\hline \multirow[t]{2}{*}{ Cou (g) } & 1 & 20 & 863 & 136 & 16 & 640 & 1080 \\
\hline & 2 & 23 & 730 & 110 & 15 & 576 & 980 \\
\hline \multirow[t]{2}{*}{ Poitrine (g) } & 1 & 20 & 1006 & 176 & 17 & 750 & 1490 \\
\hline & 2 & 23 & 1053 & 150 & 14 & 790 & 1290 \\
\hline \multirow[t]{2}{*}{ Gigot (g) } & 1 & 20 & 2187 & 283 & 13 & 1660 & 2750 \\
\hline & 2 & 23 & 2317 & 230 & 10 & 1974 & 2840 \\
\hline \multirow[t]{2}{*}{ Côtes (g) } & 1 & 20 & 1624 & 238 & 15 & 1200 & 2080 \\
\hline & 2 & 23 & 1511 & 165 & 11 & 1187 & 1820 \\
\hline
\end{tabular}

${ }^{1}$ Expérimentation : 1, selon Alexandre et coll., 2008, Livest. Res. rural Dev. ; 2, selon Archimède et coll., 2007, Small Ruminant Res. ; 3, selon Marie-Magdeleine et coll., 2007, Trop. Anim. Health Prod.

${ }^{2}$ Coefficient de variation (déviation standard / moyenne) 
symphise, profondeur de la poitrine au niveau de la sixième côte et longueur interne de la carcasse symphise pubienne - pointe du sternum. La carcasse gauche a été alors découpée en cinq morceaux déterminés par des points anatomiques précis répertoriés par la découpe standardisée de Boccard et Dumont (6) : les gigot, cou, épaule, poitrine et train de côtes ont alors été pesés.

\section{Calculs et analyses statistiques}

Le poids vif vide (PVV) a été calculé comme la différence entre le poids vif à jeun avant abattage et le contenu du tube digestif ; le rendement de carcasse vrai a été calculé comme le rapport entre le poids de carcasse froide et le poids vif vide (CF/PVV).

L'index de compacité (IC) de la carcasse est défini (18) comme étant le rapport de la largeur du bassin sur la longueur queue - cou et l'index de compacité du gigot comme étant le rapport de la largeur du bassin sur la longueur du gigot. De plus, des index pondéraux (IP) ont été calculés (poids sur longueur, de la carcasse ou du gigot).

La base de données $(n=43$ agneaux $)$ des deux expérimentations citées est décrite dans le tableau I. Trois classes de poids d'abattage ont pu être déterminées : $28 \mathrm{~kg}(26,4-29,6 \mathrm{~kg}), 32 \mathrm{~kg}$ $(30,3-33,8 \mathrm{~kg})$ et $36 \mathrm{~kg}(34,2-38,4 \mathrm{~kg})$. Une analyse de variance a été réalisée sur les différentes variables mesurées avec le modèle linéaire général (logiciel Minitab V14,2) en prenant en compte les effets du mode d'alimentation et de la classe de poids à l'abattage, et l'interaction qui a été prise en considération quand elle s'est avérée statistiquement significative.

Les équations d'allométrie ont été déterminées sur une base de données élargie (tableau I) en prenant en compte des résultats d'abattage d'agneaux OMK $(\mathrm{n}=44)(21)$ mais où seules les carcasses et épaules ont été pesées. L'équation de Huxley (16) a été utilisée, $\log \mathrm{Y}=\mathrm{a} \log \mathrm{X}+\mathrm{b}$, où $\mathrm{Y}$ est le poids de carcasse et $\mathrm{X}$ le poids à jeun ou le poids vif vide, ou encore $\mathrm{Y}$ le poids d'un morceau donné et $\mathrm{X}$ le poids de la carcasse.

\section{RESULTATS ET DISCUSSION}

Les performances de croissance des agneaux OMK, variant de 78 à $172 \mathrm{~g} / \mathrm{j}$ et pouvant atteindre jusqu'à $200 \mathrm{~g} / \mathrm{j}$, ont dépendu principalement des types de rations testées (tableau I) et ont montré aussi une grande variabilité animale (20 p. 100 de coefficient de variation). Des techniques d'embouche intensive, testées sous des latitudes africaines, aboutissent à des croissances similaires au Zimbabwe $(133 \mathrm{~g} / \mathrm{j} ; 22)$, mais aussi à des niveaux moindres $(75-80 \mathrm{~g} / \mathrm{j})$ au Burkina Faso ou au Bénin $(2,9)$, sans doute en raison de l'âge et de l'état des animaux mis à l'engrais.

\section{Carcasse et découpe}

Les poids moyens de carcasse froide (tableau II) ont augmenté logiquement avec la classe de poids vif des agneaux $(2 \mathrm{~kg}$ de plus par classe de poids en moyenne). Les rendements de carcasse (commerciaux, 45 p. 100 ; vrais, 59 p. 100) n'ont pas varié avec la gamme de poids comme déjà rapporté (30), sans doute en liaison avec des dépôts de gras limités constatés sur les carcasses $(1,5)$. Il est à noter que les rendements en carcasse ont été parmi les meilleurs des pays chauds : 44 p. 100 (15), 48 p. 100 (19, 22, 25), 50 p. 100 (10, 12), 52 p. 100 (17), ce qui est une conclusion encourageante pour ces premiers essais d'engraissement intensif des agneaux Martinik.

Les poids des différents morceaux (tableau II) ont augmenté significativement $(\mathrm{P}<0,01)$ et de façon logique avec la classe de poids de carcasse. La différence entre les deux lots extrêmes a été de 28 p. 100 pour l'épaule, 30 p. 100 pour le gigot et 40 p. 100 pour le collier. Les proportions d'épaule, de gigot, et de collier ont représenté 18,34 , et 12 p. 100 de la carcasse, sans présenter de différences significatives entre classes de poids. Les proportions des morceaux ont été semblables à celles des ovins de type viande observées par Laville et coll. (32 à 36 p. 100 pour le gigot et 16 p. 100 pour l'épaule ; 18), et des agneaux Manchega rapportées par Zurita-Juarez et coll. (31 et 19 p. 100 ; 33). Les agneaux OMK semblaient présenter des proportions de morceaux nobles dans la carcasse, supérieures aux valeurs rapportées sur des ovins tropicaux par Ramos de Siqueira et coll. (27) au Brésil, Nagalakshmi et coll. (23) en Inde, et Guttierrez et coll. (15) au Mexique, bien qu'il soit difficile de comparer les techniques de découpe.

\section{Mensurations des carcasses}

Les mensurations des carcasses (tableau III) ont montré que, avec l'alourdissement des animaux, les longueurs (quel que soit le site de mesure sur la carcasse) et la largeur de bassin augmentaient $(\mathrm{P}<0,05)$ progressivement dans la suite logique de leur gamme de poids (près de 9 p. 100 de différence entre les poids extrêmes) et la profondeur de poitrine ne variait que de 4 p. 100.

Les index de compacité de carcasse (de l'ordre de 0,32) et du gigot (de l'ordre de 0,45$)$ n'ont pas varié selon le poids, alors que les index pondéraux ont été différents $(\mathrm{P}<0,01)$. Les index pour la carcasse ont été dans la gamme présentée pour la race Manchega (IP de 214 à $291 \mathrm{~g} / \mathrm{cm}$ pour un poids de carcasse froide de 14,8 kg ; 33) mais inférieurs à ceux des agneaux français sélectionnés pour leur conformation (IC de 0,37 et IP de $300 \mathrm{~g} / \mathrm{cm} ; 13,18$ ). Cependant, ces valeurs restent proportionnelles au format et poids de carcasse des races considérées qui pèsent $4 \mathrm{~kg}$ de plus que la carcasse des OMK. Les mêmes index calculés pour le gigot ont été largement inférieurs à ceux relevés dans les études citées, les différences venant du poids et de la taille des gigots (plus de $3 \mathrm{~kg}$ et $23 \mathrm{~cm}$ comparativement à $2,2 \mathrm{~kg}$ et $18 \mathrm{~cm}$ ).

\section{Tableau II}

Effet du poids d'abattage sur les poids, rendement et notes des carcasses des agneaux Martinik alimentés dans différentes conditions ( $n=43$, données ajustées pour l'effet alimentation)

\begin{tabular}{|c|c|c|c|}
\hline $\begin{array}{l}\text { Classe de poids } \\
\text { à l'abattage (kg) }\end{array}$ & 28 & 32 & 36 \\
\hline Poids vif vide (kg) & $20,1^{\mathrm{a}}( \pm 0,6)$ & $23,5^{b}( \pm 0,3)$ & $26,9^{\mathrm{c}}( \pm 0,4)$ \\
\hline $\begin{array}{l}\text { Carcasse froide } \\
(\mathrm{kg})\end{array}$ & $11,7^{\mathrm{a}}( \pm 0,3)$ & $13,8 \mathrm{~b}( \pm 0,2)$ & $15,6^{\mathrm{c}}( \pm 0,3)$ \\
\hline $\begin{array}{l}\text { Rendement } \\
\text { de carcasse (\%) }\end{array}$ & $60,0( \pm 0,3)$ & $59,4( \pm 0,2)$ & $58,5( \pm 0,3)$ \\
\hline $\begin{array}{l}\text { Note de } \\
\text { conformation }\end{array}$ & $3,8^{\mathrm{a}}( \pm 0,2)$ & $4,1^{\mathrm{b}}( \pm 0,2)$ & $4,5^{b}( \pm 0,2)$ \\
\hline Epaule (g) & $1058^{\mathrm{a}}( \pm 33)$ & $1226^{b}( \pm 24)$ & $1360^{\mathrm{c}}( \pm 30)$ \\
\hline Collier (g) & $651^{\mathrm{a}}( \pm 27)$ & $814^{b}( \pm 20)$ & $908^{c}( \pm 25)$ \\
\hline Poitrine (g) & $880^{\mathrm{a}}( \pm 41)$ & $1029^{b}( \pm 31)$ & $1170^{\mathrm{C}}( \pm 38)$ \\
\hline $\begin{array}{l}\text { Train de côtes } \\
\text { (g) }\end{array}$ & $1334^{\mathrm{a}}( \pm 46)$ & $1594^{b}( \pm 34)$ & $1747^{c}( \pm 43)$ \\
\hline Gigot (g) & $1967^{\mathrm{a}}( \pm 49)$ & $2233^{b}( \pm 36)$ & $2549^{\mathrm{c}}( \pm 45)$ \\
\hline
\end{tabular}

a,b,c Sur une même ligne, les valeurs suivies de lettres différentes diffèrent significativement à $\mathrm{P}<0,05$ 


\section{Tableau III}

Effet du poids d'abattage sur les mensurations ${ }^{1}$ des carcasses des agneaux Martinik alimentés dans différentes conditions ( $n=43$, données ajustées pour l'effet alimentation)

\begin{tabular}{|c|c|c|c|}
\hline $\begin{array}{l}\text { Classe de poids } \\
\text { à l'abattage (kg) }\end{array}$ & 28 & 32 & 36 \\
\hline $\begin{array}{l}\text { Largeur du } \\
\text { bassin }(\mathrm{cm})\end{array}$ & $17,2^{a}( \pm 0,2)$ & $17,7^{\mathrm{a}}( \pm 0,1)$ & $18,8^{b}( \pm 0,2)$ \\
\hline $\begin{array}{l}\text { Longueur } \\
\text { queue - cou (cm) }\end{array}$ & $54,2^{a}( \pm 0,6)$ & $56,7^{b}( \pm 0,5)$ & $58,6^{b}( \pm 0,6)$ \\
\hline $\begin{array}{l}\text { Profondeur au } \\
\text { thorax }(\mathrm{cm})\end{array}$ & $26,6^{\mathrm{a}}( \pm 0,3)$ & $27,1^{\mathrm{a}}( \pm 0,3)$ & $27,8^{b}( \pm 0,3)$ \\
\hline $\begin{array}{l}\text { Longueur du } \\
\text { gigot }(\mathrm{cm})\end{array}$ & 38,2 a $( \pm 0,4)$ & $39,8^{b}( \pm 0,3)$ & $40,8^{b}( \pm 0,4)$ \\
\hline $\begin{array}{l}\text { Longueur de } \\
\text { carcasse interne } \\
\text { (cm) }\end{array}$ & $61,1^{\mathrm{a}}( \pm 0,4)$ & $63,4{ }^{b}( \pm 0,3)$ & $66,4^{\mathrm{C}}( \pm 0,8)$ \\
\hline $\begin{array}{l}\text { Index de } \\
\text { la carcasse } \\
\text { (largeur/longueur) }\end{array}$ & $0,32( \pm 0,004)$ & ) $0,31( \pm 0,003)$ & ) $0,32( \pm 0,004)$ \\
\hline $\begin{array}{l}\text { Index du gigot } \\
\text { (largeur/longueur) }\end{array}$ & $0,45( \pm 0,006)$ & $0,44( \pm 0,005)$ & ) $0,46( \pm 0,006)$ \\
\hline $\begin{array}{l}\text { Index pondéral } \\
\text { carcasse (poids/ } \\
\text { longueur) (g/cm) }\end{array}$ & $214^{\mathrm{a}}( \pm 4)$ & $244^{b}( \pm 4)$ & $268^{c}( \pm 5)$ \\
\hline $\begin{array}{l}\text { Index pondéral } \\
\text { gigot (poids/ } \\
\text { longueur) (g/cm) }\end{array}$ & $51^{\mathrm{a}}( \pm 1)$ & $56^{b}( \pm 1)$ & $62^{\mathrm{c}}( \pm 1)$ \\
\hline
\end{tabular}

${ }^{1}$ Selon la nomenclature officielle de Boccard et Dumont, 1955, Ann. Zootech. a,b,c Sur une même ligne, les valeurs suivies de lettres différentes diffèrent significativement à $\mathrm{P}<0,01$

\section{Equations d'allométrie}

Le coefficient d'allométrie de la carcasse relative au poids à jeun (tableau IV) a varié de 0,105 point par rapport à l'allométrie relative au poids vif vide. Le premier cité a été supérieur à 1 (la CF aurait un développement plus tardif que celui du poids à jeun) et le coefficient calculé par rapport au poids vif vide a été inférieur à 1 (la CF serait plus précoce que le PVV). Il est vrai que, entre les deux estimateurs $\mathrm{X}$ du coefficient d'allométrie, la différence vient du tube digestif qui est connu pour être tardif par rapport aux abats rouges. Ce qui va dans le sens d'une conclusion justifiée depuis longtemps par Boccard et Dumont (7) qui soulignent l'importance des calculs à partir du PVV pour les ruminants (afin de se défaire des variations du contenu digestif et des poids relatifs des compartiments du tube digestif).

Les coefficients d'allométrie de l'épaule par rapport à la CF ont été de niveaux différents selon la base de données considérée (tableau IV), sans doute en relation avec l'étendue de la gamme de variation du poids de CF, comme l'ont souligné Boccard et Dumont (7) qui indiquent que les poids relatifs des morceaux nobles diminuent sensiblement d'une gamme de poids à une autre. Dans la présente étude, les valeurs de CF de la base élargie (avec une moyenne et un minimum de 9,3 et 5,6 kg) ont été inférieures de $4 \mathrm{~kg}$ à celles de la base initiale. Aparicio-Ruiz et coll. (4) citent une valeur de coefficient d'allométrie de l'épaule bien moindre, de 0,669 , pour

\section{Tableau IV}

Croissance relative des différents morceaux de la découpe de carcasse des agneaux Martinik. Equation allométrique $\log y=a * \log x+b$

\begin{tabular}{|c|c|c|c|c|c|}
\hline Variable y & $\mathbf{n}$ & a & b & SE & $\mathbf{R}^{2}$ \\
\hline \multicolumn{6}{|c|}{ Données relatives au poids à jeun (x) } \\
\hline Carcasse & 87 & 1,059 & $-0,461$ & 0,027 & 94,7 \\
\hline \multicolumn{6}{|c|}{ Données relatives au poids vif vide $(\mathrm{x})$} \\
\hline Carcasse & 87 & 0,906 & $-0,109$ & 0,027 & 94,7 \\
\hline Epaule & 87 & 0,716 & 2,103 & 0,033 & 78,2 \\
\hline \multicolumn{6}{|c|}{ Morceaux relatifs au poids de carcasse $(\mathrm{x})$} \\
\hline \multirow[t]{2}{*}{ Epaule } & 87 & 0,820 & $-0,306$ & 0,020 & 92,1 \\
\hline & 43 & 0,915 & $-0,704$ & 0,019 & 85,5 \\
\hline $\mathrm{Cou}$ & 43 & 1,042 & $-1,415$ & 0,052 & 53,0 \\
\hline Poitrine & 43 & 1,025 & $-1,231$ & 0,041 & 63,5 \\
\hline Gigot & 43 & 0,891 & $-0,334$ & 0,020 & 84,7 \\
\hline
\end{tabular}

des carcasses d'agneaux légères de 10-12 kg, ce qui soutiendrait la présente hypothèse.

Il est déjà bien établi que la croissance des différentes unités anatomiques n'est pas isométrique. Les résultats de cette étude préliminaire ont montré que l'agneau OMK n'échappait pas à la règle. En concordance avec de nombreux travaux $(7,28,33)$ les gigots et épaules ont eu des allométries inférieures à 1 , soit un développement proportionnellement moins rapide que le gain de poids de carcasse. En revanche, les observations de cette étude ne sont pas en concordance avec les travaux cités pour les coefficients d'allométrie du collier et de la poitrine qui sont supérieurs à 1 . Ceci pourrait être lié à des différences génétiques, rapportées chez des animaux croisés européens et ovins à poils du Brésil (14).

\section{CONCLUSION}

La plupart des caractéristiques des carcasses d'agneaux Martinik engraissés intensivement ont semblé comparables, toutes proportions gardées (relatives au poids de carcasse), à celles de génotypes à viande. Cependant, les index linéaires et pondéraux des gigots ont été bien moindres et s'expliquent par le fait que l'OMK n'est pas encore sélectionné sur ce critère. Ces premières variables descriptives de carcasse se classent parmi les meilleures données d'ovins tropicaux élevés en conditions favorables.

Les paramètres de découpe et de mensurations de carcasse d'ovin Martinik mériteraient d'être d'avantage évalués dans des études ultérieures afin d'élargir la gamme de variation. L'accumulation de données factuelles sur la description des carcasses de l'OMK pourrait être mise à disposition des acteurs de la filière afin de communiquer sur les critères de sélection intra race et/ou les choix de systèmes d'élevage. Par ailleurs, pour les filières locales, il convient de souligner que les carcasses de moutons à poils, engraissés intensivement, s'alourdiraient de façon conséquente (jusqu'à $16 \mathrm{~kg}$ ) en présentant, par ailleurs, une note de conformation voisine de 4 (grille agneaux légers), jugée comme un bon niveau. Tout ceci confirmerait l'intérêt de la production d'agneaux lourds. 


\section{Remerciements}

Les auteurs tiennent à remercier C. Anais, P. Despois, G. Gravillon, F. Silou et W. Troupé pour leurs apports techniques, ainsi que les responsables des filières Amiv et Iguavie. Les travaux ont été réalisés grâce au financement de la région Martinique, du Fonds européen d'orientation et de garantie agricole (Feoga) et de l'Office de développement de l'économie agricole des départements d'OutreMer (Odeadom).

\section{BIBLIOGRAPHIE}

1. ALEXANDRE G., COPPRY O., BOCAGE B., FLEURY J., ARCHIMEDE H., 2008. Effect of live weight at slaughter on the carcass characteristics of intensively fattened Martinik sheep fed sugar cane supplemented with pea flour. Livest. Res. rural Dev., 20.

2. ALKOIRET T.I., MANNE A.A.S., GBANGBOCHE A.B., ATTAKPA E.Y., 2007. Fattening performance of Djallonke sheep supplemented with cotton seed husks in Benin. Livest. Res. rural Dev., 19.

3. AMEGEE Y., 1984. Le mouton de Vogan (croisé Djallonké x Sahélien) au Togo III. Performances d'engraissement et rendement des carcasses. Revue Elev. Méd. vét. Pays trop., 37 : 97-106.

4. APARICIO RUIZ F., TOVAR ANDRADA J., GARCIA PALOMA J.A., 1982. Coeficiente de alometria en las diferentes piezas de canales de corderos machos de raza Merina Campineza. Arch. Zootec., 120: $205-210$

5. ARCHIMEDE H., ALEXANDRE G., PELLONDE P., DESPOIS P., BOCAGE B., ETIENNE T., 2007. Growth performances and carcass traits of Ovin Martinik lambs fed various ratios of tropical forage to concentrate under intensive conditions. Small Ruminant Res., 75: 162-170.

6. BOCCARD R., DUMONT B.L., 1955. Etude de la production de viande chez les ovins. I. La coupe des carcasses. Définition d'une coupe de référence. Ann. Zootech., 3 : 241-257.

7. BOCCARD R., DUMONT B.L., 1976. La qualité des carcasses ovines. In : Proc. $2^{\text {es }}$ journées de la Recherche ovine et caprine, croissance, engraissement et qualité des carcasses d'agneaux et de chevreaux. Paris, France, Inra-Itovic, p. 44-78.

8. BOURZAT D., BONKOUNGOU E., RICHARD D., SANFO R., 1987. Essais d'intensification de la production animale en zone sahélosoudanienne : alimentation intensive de jeunes ovins dans le Nord du Burkina. Revue Elev. Méd. vét. Pays trop., 40 : 151-156.

9. BULDGEN A., DETIMMERMAN F., PIRAUX M., COMPERE R., 1992. Les techniques $d^{\prime}$ embouche de moutons en région soudano-sahélienne sénégalaise. Revue Elev. Méd. vét. Pays trop., 45 : 321-328.

10. CHIKHI A., BOUJENANE I., 2003. Performances de reproduction et de production des ovins de race Boujaâd au Maroc. Revue Elev. Méd. vét. Pays trop., $\mathbf{5 6}:$ 83-88.

11. COLOMER-ROCHER F., 1988. COLOMER-ROCHER F., DELFA R., SIERRA I., 1988. Método normalizado para el estudio de los caracteres cuantitativos y cualitativos de las canales ovinas producidas en el área mediterránea, según los sistemas de producción. Cuadernos INIA, 17: 19-41.

12. EWNETU ERNIAS, ALEMU YAMI, REGE J.E.O., 2006. Slaughter characteristics of Menz and Horro sheep. Small Ruminant Res., 64: $10-15$

13. FLAMANT J.C., PERRET G., 1976. Le croisement et la production de viande d'agneaux; comparaison et sélection des races de mâles. In : Proc. 2es journées de la Recherche ovine et caprine, croissance, engraissement et qualité des carcasses d'agneaux et de chevreaux. Paris, France, Inra-Itovic, p. 110-134.

14. FURUSHO-GARCIA I.F., OLALQUIAGA PEREZ I.R., BONAGURIO S., LEAL DOS SANTOS C., 2006. Alometric study of cuts and tissues of the carcass of purebred and crossbred Santa Ines lambs. Rev. Bras. Zootec., 35: 1416-1422.
15. GUTIERREZ J., RUBIO M.S., MENDEZ R.D., 2005. Effects of crossbreeding Mexican Pelibuey sheep with Rambouillet and Suffolk on carcass traits. Small Ruminant Res., 70: 1-5.

16. HUXLEY J.S., 1932. Problems of relative growth. London, UK, Metheun, $276 \mathrm{p}$.

17. KAWAS J.R., GARCIA C.R., GARZA C.F., FIMBRES D.H., OLIVARES S.E., HERNANDEZ V.G., LU C.D., 2007. Effects of sodium bicarbonate and yeast on nutrient intake, digestibility, and ruminal fermentation of light-weight lambs fed finishing diets. Small Ruminant Res., 67: 157-163.

18. LAVILLE E., BOUIX J., SAYD T., EYCHENNE F., 2002. La conformation bouchère des agneaux. Etude d'après la variabilité génétique entre races. Prod. Anim., 15: 53-56

19. MAHGOUB O., LU C.D., EARLY R.J., 2000. Effects of dietary energy density on feed intake, body weight gain and carcass chemical composition of Omani growing lambs. Small Ruminant Res., 37: 35-42.

20. MAHIEU M., AUMONT G., ALEXANDRE G., 1997. Elevage intensif des ovins tropicaux à la Martinique. Prod. Anim., 10 : 21-32.

21. MARIE-MAGDELEINE C., LIMEA L., DESPOIS P., SAMINADIN G., ARCHIMEDE H., ALEXANDRE G., 2008. Growth and carcass traits of Martinik sheep fed Musa paradisia in replacement of Dichantium hay. Trop. Anim. Health Prod. (sous presse)

22. MATIKA O., VAN WYK J.B., ERASMUS G.J., BAKER R.L., 2003. A description of growth, carcass and reproductive traits of Sabi sheep in Zimbabwe. Small Ruminant Res., 48: 119-126.

23. NAGALAKSHMI D., SASTRY V.R.B., KESAVA RAO V., 2002. Influence of feeding processed cottonseed meal on meat and wool production of lambs. Asian-Aust. J. Anim. Sci., 15: 26-33.

24. NAVES M., ALEXANDRE G., LEIMBACHER F., MANDONNET N., MENENDEZ BUXADERA A., 2001. Le point sur les programmes de gestion des ressources génétiques chez les espèces de ruminants dans la Caraïbe. Prod. Anim., 14 : 182-192.

25. NEGUSIE E., ROTTMAN O.J., PIRCHNER F., REGE J.E.O., 2004 Growth and carcass composition of tropical fat-tailed Menz and Horro sheep breeds. Anim. Sci., 78: 245-252.

26. OfIVAL, 2005. Classifications des ovins. Guide technique et réglementaire; pesée, classement, marquage. Montreuil sous Bois, France, Office de l'élevage. http://www.ofival.fr/guide-pcm-ext/pageweb/p-31a34.htm (09/01/05).

27. RAMOS DE SIQUEIRA E., DEODATO SIMOES C., FERNANDES S., 2001. Sex and slaughter weight effects on meat production of lambs. Carcass morphometric evaluation, cut weights, tissues and offals percentages. Rev. Bras. Zootec., 30: 1299-1307.

28. RUIZ DE HUIDOBRO F., CANAQUE V., 1994. Produccion de carne de corderos de raza Manchega. $\mathrm{V}$. Crecimiento relativo del quinto cuarto y de los tejidos y piezas de la canal. Invest. Agr. Prod. Sanid. Anim., 9: 95-108.

29. SANTOS-SILVA J., MENDES I.A., BESSA R.J.B., 2002. The effect of genotype, feeding system and slaughter weight on the quality of light lambs. 1. Growth, carcass composition and meat quality. Livest. Prod. Sci., 76: 17-25.

30. SANTOS-SILVA J., VAZ PORTUGAL A., 1991. Contribution to the study of body development in Merino prococe lambs, subject to two diets. Livest. Res. rural Dev., 3: http://www.cipav.org.co//rrd//rrd3/2/ portugal.htm

31. VERTUEUX C., MANDONNET N., LEIMBACHER F., ANTOINE S. DOMARIN D., 2006. Potentiel de production du mouton Martinik : une contribution possible à l'intensification de l'agriculture caribéenne. In : Proc. $7^{\mathrm{e}}$ journée technique de l'Amadepa, Martinique, 26 avril 2006. Le Lamentin, Martinique, Amadepa, p. 118-123.

32. WILDEUS S., Ed., 1991. Hair sheep research symposium. St Croix, USA, University of the Virgin Islands, $362 \mathrm{p}$.

33. ZURITA JUAREZ M.C., VERA Y VEGA A., APARICIO RUIZ F., 1980. Contribucion al studio y a la tipificacion de las canales de corderos de raza Manchega. Arch. Zootec., 114: 139-190.

Reçu le 13.12.2007, accepté le 16.09.2008 


\section{Summary}

Alexandre G., Bocage B., Coppry O., Weisbecker J.L., Mahieu $\mathbf{M}$., Archimède $\mathbf{H}$. Parameters of Carcass Cuts and Measurements of Martinik Lambs Managed under Intensive Conditions

The Martinik sheep is selected for its good adaptive and reproductive traits. The production sector reproaches it with low carcass conformation, although its carcasses have been little described. Two intensive fattening experiments were conducted, whereby lambs were slaughtered for linear measurements and cuts. The variables were analyzed and allometric equations were determined. The database $(n=43)$ was adjusted for feeding levels and slaughter weight groups (SW: 28, 32 and $36 \mathrm{~kg}$ ). Mean cold carcass (CC) weights varied $(\mathrm{P}<0.05)$ from 11.7 to $15.6 \mathrm{~kg}$. Carcass yields were on average $59 \%(P>0.05)$. The weights of all carcass cuts significantly $(P<0.01)$ increased with $C C$ increase. Shoulders and legs represented 18 and $34 \%$ of CC, respectively. Various measurements taken on the carcass and pelvis width significantly increased $(P<0.05)$ with $C C$ increase (near 9\% difference between extreme weights). Indices of carcass and leg compactness (0.32 and 0.45 , respectively) did not vary with SW, whereas weight indices did. Allometric coefficients of the shoulder (0.915) and leg (0.891) were in line with those of the literature. This preliminary description of Martinik sheep carcasses could be made available to the sector actors in order to pursue the work and help to better select breeds and management systems.

Keywords: Martinik sheep - Carcass - Body measurement Body conformation - Growth - French West Indies.

\section{Resumen}

Alexandre G., Bocage B., Coppry O., Weisbecker J.L., Mahieu $\mathbf{M}$., Archimède $\mathbf{H}$. Parámetros de corte y medidas de las carcasas de corderos Martinik criados bajo condiciones intensivas

El ovino Martinik se selecciona por sus capacidades de adaptación y su productividad. Las filiales le reprochan una conformación de carcasa baja, a pesar de que estas carcasas han sido poco descritas. Se realizaron sacrificios durante dos prácticas de engorde intensivo (estudiadas) y se registraron los cortes y las medidas lineares. Se analizaron estas variables y se llevó a cabo la determinación de las ecuaciones de alometría. La base de datos ( $\mathrm{n}=43$ corderos) se codificó para los niveles de alimentación y la clase de peso al sacrificio (PS: 28,32 y $36 \mathrm{~kg}$ ). Los pesos medios de la carcasa fresca (CF) variaron $(P<0,05)$ de 11,7 a $15,6 \mathrm{~kg}$. Los rendimientos de la carcasa fueron en promedio de $59 \%(\mathrm{P}>0,05)$. Los pesos de los diferentes cortes aumentaron significativamente $(P<0,01)$ con la gama de CF. Las proporciones de la espalda y de la pierna representaron 18 y $34 \%$ respectivamente de la CF. Las diferentes longitudes medidas sobre la carcasa y la longitud de la pelvis aumentaron $(\mathrm{P}<0,05)$ con la CF (cerca de $9 \%$ de diferencia entre los pesos extremos). Los índices de compacte de la carcasa $(0,32)$ y de la pierna $(0,45)$ no variaron según la PS, mientras que los índices ponderales fueron diferentes. Los coeficientes de alometría de la espalda $(0,915)$ y de la pierna $(0,891)$ fueron de la misma importancia que los de la literatura. Estas primeras descripciones de las carcasas del ovino Martinik podrían proponerse a la filial con el fin de continuar el trabajo y dar mejor base a la escogencia de razas y sistemas.

Palabras clave: Ovino Martinik - Canal animal - Medición del cuerpo - Conformación animal - Crecimiento - Antillas francesas. 